associated with globe trouble, such as unilateral scleritis and uveitis or cellulitis. If there is no obvious disease the smallest exploratory procedure compatible with an accurate diagnosis is indicated. I have seen no bad results from a careful extension of the sphenoidal ostium downwards, and at least it precludes retention in this all-important space. Indis. criminate cutting operations in the nose are without doubt almost as criminal as is a wholesale extraction of teeth on account of a suspected focal infection.

'There are so many points in connexion with optic atrophy which interest the physician that it is difficult to pick out types for discussion. As regards general paralysis, it has been suggested to me by an eminent mental specialist that general paralysis is often missed in India. In this city 1 rarely see it. In the following two cases there was practically complete loss of vision in association with extensive central nervous system syphilis of a congenital type.

In one the partial atrophy is of a decidedly post-neuritic type, in the other it is of a primary or simple type. In the first case there was extensive neuritis and extensive paralyeis. When we first saw this boy he was blind except for perception of light. Colonel Elwes, first physician, General Hospital, kindly took over the case. The patient is now able to see perfectly well and can get about. He has contraction of the fields, a slight degree of optic atrophy, but gets $6 / 5$ central vision in each eye. He has a certain amount of spastic paratysis of the limbs, he is slightly deaf, has not had interstitial keratitis, but has the characteristic teeth.

In the other case the atrophy is of a simple variety, there has never been papillitis, the gait is of a spastic type, reflexes are jucreased, and vision is markedly reduced. The teeth are not in any way typical, but the two other members of Hutchinson's triad are present - the deafness and remains of an interstitial keratitis.

Finally, I mention the association of optic atrophy with the meningitides of childhood. We very frequently see double optic atrophy in children, either simple or post-papillitic, and as often as not we get a history of fever or fits preceding the onset of bad vision. The history is unreliable, and as in this hospital we do not come in much contact with the diseases of children, I would be very glad to obtain the views of general practitioners or plysicians on the subject of meningitis occurring in children in which recovery takes place but which is followed by optic atrophy.

\section{DIPHTHERIA CARRIERS.}

\section{BY}

\author{
Lievt.-Colonel F. W. SUMNER, M.D.Chntab., \\ F.R.C.S.EDIN., \\ INDIAN MED'CAL SERVICE.
}

As outbreak of diphtheria occurred at a school in Simla in March, 1922. Eight cases occurred from March 14th to 23rd : they were all from the same source of infection-a boy who arrired from Karachi in March suffering from diphtheria; the others were direct contacts.

Sporadic cases occurred on the following dates: May 19th, 1; July 3rd, 1; July 7th, 1; September 9th to 19th, 4 . There were 15 cases in all with one death (case of May 19th).

The interest in this outbreak lies in the question of carriers and what factors go to make "healthy carriers" as apart from "disease carriers." By the former I mean those who have shown no symptoms of the disease, and yet who have present in their throats diphtheria bacilli; by the "disease carrier" I mean those who have had a definite attack of diphtheria and have entirely recovered except that throat swabs still show the presence of diphtheria bacilli.

In this outbreak the whole school of 200 boys were given, in March, prophylactic injections of diphtheria antitoxin, and, later, as cases occurred, all contacts were given further proplyylactic injections.

In the first batch of 200 there were roughly 5 per cent. who, after some days, developed mild anaphylactic symptoms (lashes and painful joints); after the subsequent injections this percentage increased to roughly 10 per cent., with rather more pronounced symptoms, but nothing of the nature of anaphylactic shock, nor, indeed, any such symptoms as to cause a moment's anxiety.

On May 20th examination for "disease carriers," by taking three consecutive daily swabs instead of the usual one from each of the eight cases who had recovered from diphtheria, showed one to be an intermittent carrier. On the occurrence of the cases in July a furlher examination of these cases, the three swabs being increased to six consecutive daily swabs, showed that six out of the original eight were intermittent carriers: these were duly segregated.

On September 9th, after two months' interval, another sporadic case occurred; all possible disease carrieis-on the six day test-had been segregated; there was no other known source of infection. 'The health officer and I decided that it would be to the interest of the boys, in the presence of this unknown source of infection, to advise the governors to close school ; we were of opinion that the school drains had nothing to do with the infection.

I decided to examine the throats of all the schoolboss and a!so the menial establishment with their families, and to mark down for bacteriological examination all those who had reddened throats or large tonsils. There were 59 such cases; one was an ambulatory case of mild diphtheria, both tonsils being covered by a creamy membrane; he was unaware that he was ill; his throat swab was positive; he had the signs and symptoms of a mild diphtheria. Of the remaining 58 cases 50 per cent. proved to be carriers. A few of the remaining 141 boys were examined as these had to remain behind for an examination, the remainder returning to their homes.

Thus one may say that of the 200 boys 15 contracted diphtheria, and out of the remainder (plus 14 of these) 80 were examined and 40 found to be carriers. Some 250 prophylactic injections of diphtheria antitoxin were given.

What are the facts of this condition of things? The drains had been the same for twenty years and no such epidemic had occurred before; the source of infection was the boy from Karachi; prophylaxis had been done on a large scale; an injection of diphtheria antitoxin neutralizes some or all of the toxins of any diphtheria bacilli present and thus prevents more or less the symptoms of diphtheria arising, but does not kill the germs that may be present-diphtheria bacteriolysin would be necessary for that purpose, but is not available in India.

We are driven to the conclusion that prophylaxis is a twoedged sword. We must, on the outbreal of diphtheria, give prophylactic injections to all contacts, but where antitoxin only is used.it behoves us to be on the look-out for a crop of diphtheria carriers as a result. A recent bacteriological examination ${ }^{1}$ carried out at the Royal Hospital for Sick Children and the school clinics, Liverpool, on a series of tonsils removed at that hospital, showed that in 30 per cent. diphtheroid bacilli were present. It is very probable that there is quite a considerable percentage of the public at large who are diphtheroid carricrs.

From a clinical point of view there are three types of cases of "sore throat" : (1) Where the clinical manifestations of a diphtheritic throat are present and where the bacteriological report is positive. (2) Where the clinical manifestations of a diphtheritic throat are present but where the bacteriological report is negative. (3) Where there are no clinical manifestations of a diphtheritic throat and the bacteriological report is positive.

Classes (1) and (2) must both be regarded as diphtheria and treated accordingly. It is of interest that of the 15 cases of diphtheria which occurred during this outbreak there were two pronounced negative bacteriologically, and one of these was the only fatal case of the series; there was no error in technique in putting up the throat swab or delay in incubation of a smear from it.

As regards class (3) it is possible and probable that such cases are merely some inflammatory condition of the nasopharynx, and the bacilli found are non-pathogenic and noncausative of the condition; clinically there were several such cases in this series, which were nevertheless looked on and treated as diphtheritics.

1N. Wall . The Bactorio of REFERENCE.

1N. Wall : The Bacteria of the Tonsils and Adenoids, BRITISH MEDICAT

THE Gifford lectures delivered in the University of Aberdeen in 1921 and 1922 by Dr. E. W. Hobson, F.R.S., Sadleirian Professor of Pure Mathematics in the University of Cambridge, will be published in a volume this summer by the Cambridge University Press. In the lectures an attempt was made to settle the relation of " that complex of knowledge and ideas which is denoted by the term Natural Science" to religion and philosophy at the present day. The author has examined the historical development, aims, and true characteristics of the various departments of natural science, and arrives at the conclusion that many men of science have exaggerated our dependence on natural science in our general outlook on the world and in present-day thought. 